\title{
Early goal-directed therapy versus usual care in the management of septic shock
}

\author{
Reviewed by: Michael Gottlieb, MD*
}

Clinical question

Does early goal-directed therapy decrease mortality when compared with usual care?

Article chosen

Angus DC, Barnato AE, Bell D, et al. A systematic review and meta-analysis of early goal-directed therapy for septic shock: the ARISE, ProCESS and ProMISe Investigators. Intensive Care Med 2015;41(9):1549-60. doi:10.1007/s00134-015-3822-1.

Keywords: Early Goal Directed Therapy, EGDT, Sepsis, Septic Shock, Usual Care

\section{OBJECTIVE}

Prior meta-analyses demonstrated a potential survival benefit for early goal-directed therapy (EGDT) in comparison with usual care but were limited by methodological flaws and the inclusion of predominately nonrandomized trials. ${ }^{1-3}$ In light of numerous, large, multicentre, randomized controlled trials (RCTs) recently published, the authors published a meta-analysis comparing EGDT with usual care exclusively among RCTs assessing for differences in mortality rates.

\section{BACKGROUND}

Sepsis is a leading cause of death in the United States. ${ }^{4}$ In 2001, Rivers et al. first described a specific early goal-directed therapy (EGDT) protocol to reduce mortality among emergency department patients presenting with severe sepsis or septic shock. ${ }^{5}$ This EGDT protocol was subsequently adopted by many providers and incorporated into numerous guidelines, including the Surviving Sepsis Campaign. ${ }^{6}$ However, several recent randomized controlled trials (RCTs) have challenged whether a survival benefit exists compared to current usual sepsis care. ${ }^{7-9}$ In contrast to previous meta-analyses, this systematic review and meta-analysis assessed the mortality difference between EGDT and usual care exclusively among RCTs. ${ }^{1,2}$

\section{SUMMARY OF METHODS}

Two authors independently searched PubMed, EMBASE, and the Cochrane Central Register of Controlled Trials from January 2000 to January 2015. The authors also searched clinical trial registries and contacted experts in the field to identify additional unpublished studies. Only RCTs comparing EGDT with either usual care or another resuscitation strategy that did not incorporate EGDT were included. The primary outcome was mortality among patients initially treated in the emergency department. Secondary outcomes included intensive care unit (ICU) admission rates, duration of stay in the ICU, and duration of stay in the hospital. There were no language restrictions. Two authors independently extracted data, and differences of opinion were resolved by consensus. Two independent assessors who were not involved in the conduct of any included studies assessed risk of bias with the Cochrane Collaboration tool with differences of opinion resolved by consensus. A fixed-effect model was used to obtain an estimate of the effect size of both the primary and secondary outcomes expressed as pooled odds ratios (OR) with $95 \%$ confidence interval (CI), which was presented as a forest plot. ICU and hospital length of stay were assessed with weighted mean difference

From the *Department of Emergency Medicine, Cook County Stroger Hospital, Chicago, IL.

Correspondence to: Dr. Michael Gottlieb, Cook County Stroger Hospital, Department of Emergency Medicine, 1900 W. Polk St., 10th Floor, Chicago, IL, 60612, United States; Email: michaelgottliebmd@gmail.com 


\begin{tabular}{|c|c|c|c|c|}
\hline Study (date) & Total patients & EGDT mortality & Control group mortality & OR $(95 \% \mathrm{Cl})$ \\
\hline Rivers et al. $(2001)^{5}$ & 263 & $29.2 \%$ & $44.4 \%$ & $0.52(0.31-0.86)$ \\
\hline Jones et al. $(2010)^{11}$ & 300 & $22.7 \%$ & $16.7 \%$ & $1.47(0.82-2.60)$ \\
\hline ProCESS Investigators $(2014)^{7}$ & 1341 & $21.0 \%$ & $18.5 \%$ & $1.17(0.88-1.55)$ \\
\hline ARISE Investigators $(2014)^{8}$ & 1588 & $18.6 \%$ & $18.8 \%$ & $0.98(0.76-1.26)$ \\
\hline ProMISe Investigators $(2015)^{9}$ & 1243 & $29.5 \%$ & $29.2 \%$ & $1.02(0.80-1.30)$ \\
\hline Overall & 4735 & $23.2 \%$ & $22.4 \%$ & $1.01(0.88-1.16)$ \\
\hline
\end{tabular}

(WMD) with 95\% CI. A sensitivity analysis was conducted using a random-effects model. Heterogeneity was assessed with the $I^{2}$ statistic. Publication bias was assessed with funnel plots and the Egger's test.

\section{SUMMARY OF RESULTS}

Five studies comprising 4735 patients were included in the final analysis. Most studies had a low risk of bias across all parameters with the exception of "blinding," for which all studies had a high risk of bias. Mortality was $23.2 \%$ in the EGDT group and $22.4 \%$ in the control group, resulting in a pooled OR of $1.01(95 \%$ CI 0.88-1.16) (Table 1). EGDT was associated with increased ICU admission (OR: 2.19 [95\% CI 1.822.65]). However, there was no difference in ICU length of stay (WMD: -0.02 [95\% CI -0.47 to 0.43$]$ ) or hospital length of stay (WMD: -0.28 [95\% CI -1.18 to $0.62])$. There was a moderate degree of heterogeneity $\left(I^{2}=57 \%\right)$. Sensitivity analysis using a random-effects model did not alter the results.

\section{COMMENTARY}

In contrast to prior meta-analyses of varying methodologies, this systematic review of only RCTs demonstrated no significant difference in mortality between EGDT and usual care. ${ }^{1-3}$ There are several reasons for the difference between this and prior systematic reviews, including improved quality of the included studies, significantly larger sample sizes, and changes in the recognition and management of sepsis. Early sepsis trials demonstrated a significant mortality benefit for EGDT but were limited by small sample sizes, nonrandomized study designs, and incomplete adherence to protocols. The larger and more recent RCTs have all demonstrated no significant difference in mortality, which may be due to improved sepsis recognition, early antibiotic administration, and aggressive fluid resuscitation. ${ }^{7-9}$ Moreover, there has been increasing controversy regarding the added value of some of the EGDT components, specifically central venous oxygen saturation, dobutamine administration, and red blood cell transfusions. ${ }^{10,11}$ It is important to note that several of the authors of this meta-analysis were also lead authors of three of the included trials ${ }^{7-9}$; however, the authors used structured tools, and analyses were performed by independent assessors. Additionally, there was moderate heterogeneity across trials with slight variations in protocols and mortality outcome timing. Finally, it is important to note that all of the more recent trials included early antibiotics and aggressive resuscitation, which remain critical aspects of sepsis management.

\section{CONCLUSION}

This systematic review and meta-analysis of only RCTs demonstrated no significant difference in mortality between EGDT and usual care. However, EGDT was associated with increased admission to the ICU. These findings do not support the systematic use of protocolized EGDT for all patients with severe sepsis or septic shock.

Competing interests: None declared.

\section{REFERENCES}

1. Barochia AV, Cui X, Vitberg D, et al. Bundled care for septic shock: an analysis of clinical trials. Crit Care Med 2010;38(2):668-78.

2. Wira CR, Dodge K, Sather J, et al. Meta-analysis of protocolized goal-directed hemodynamic optimization for the management of severe sepsis and septic shock in the emergency department. West $\mathcal{F}$ Emerg Med 2014;15(1):51-9.

3. Gu WJ, Wang F, Bakker J, et al. The effect of goal-directed therapy on mortality in patients with sepsis-earlier is 
better: a meta-analysis of randomized controlled trials. Crit Care 2014;18(5):570.

4. Mayr FB, Yende S, Angus DC. Epidemiology of severe sepsis. Virulence 2014;5(1):4-11.

5. Rivers E, Nguyen B, Havstad S, et al. Early goal-directed therapy in the treatment of severe sepsis and septic shock. $N$ Engl $\mathcal{F}$ Med 2001;345(19):1368-77.

6. Dellinger RP, Levy MM, Rhodes A, et al. Surviving sepsis campaign: international guidelines for management of severe sepsis and septic shock: 2012. Crit Care Med 2013; 41(2):580-637.

7. ProCESS Investigators. A randomized trial of protocolbased care for early septic shock. N Engl 7 Med 2014; 370(18):1683-93.
8. ARISE Investigators. Goal-directed resuscitation for patients with early septic shock. $N$ Engl 7 Med 2014; 371(16):1496-506.

9. Mouncey PR, Osborn TM, Power GS, et al. Trial of early, goal-directed resuscitation for septic shock. $N$ Engl 7 Med 2015;372(14):1301-11.

10. Chung KP, Chang HT, Huang YT, et al. Central venous oxygen saturation under nonprotocolized resuscitation is not related to survival in severe sepsis or septic shock. Shock 2012;38(6):584-91.

11. Jones AE, Shapiro NI, Trzeciak S, et al. Lactate clearance vs central venous oxygen saturation as goals of early sepsis therapy: a randomized clinical trial. $7 A M A$ 2010;303(8): 739-46. 